\title{
Amoxyclav Resistance Pattern and Aerobic Bacterial Profile in Diabetic Foot Infection Patients in Bangladesh
}

\author{
Tasmia Islam ${ }^{1}$, Md. Reaz Morshed ${ }^{2}$, Farha Matin Juliana ${ }^{3}$, Fahad Hossain Palash ${ }^{4}$, Tahmina Akhter ${ }^{4}$, \\ Sonia Akter ${ }^{4}$, Mohammad Asaduzzaman*1 \\ 1.Department of Genetic Engineering and Biotechnology, Jashore University of Science and Technology, \\ Jashore, Bangladesh \\ 2.Department of Biochemistry and Molecular Biology, Noakhali Science and Technology University, Noakhali, \\ Bangladesh \\ 3.Department of Biochemistry and Molecular Biology, Jahangirnagar University, Dhaka, Bangladesh \\ 4.Department of Biochemistry, Primeasia University, Dhaka, Bangladesh
}

\begin{abstract}
Introduction: The goal of this study was to determine the frequency of bacterial isolates cultured from diabetic foot infections and to assess their amoxyclav resistance and susceptibility.Methods: A total of 378 diabetic foot lesions were included in this prospective analysis. The antibiotic susceptibility pattern of bacteria isolated from foot lesions was assessed using the Kirby-Bauer disk diffusion method.Results: The most commonly isolated Gram-positive bacteria were Staphylococcus aureus, followed by Enterococcus spp. and CoNS. The most commonly isolated Gram-negative bacteria were Klebsiella spp., Pseudomonas spp., Proteus spp., Escherichia coli, Enterobacter spp., Citrobacter spp., Citrobacter freundii, and Proteus vulgaris. Amoxyclav was found to be 100.00\% resistant against Pseudomonas aeruginosa and followed by Enterococcus spp. (89.50\%), Proteus spp. (87.50\%), Staphylococcus aureus (84.30\%), Escherichia coli $(81.50 \%)$, Klebsiella spp. $(70.50 \%)$ and Enterobacter spp. (69.20\%).Conclusion: The present study confirmed the prevalence of amoxyclav drug resistant pathogens $(84.90 \%)$ in diabetic foot ulcers. The diverse bacteria infecting the wound must be evaluated, as well as the antibiotic susceptibility patterns of the isolates from the infected lesion. This information is critical for selecting the right medications, eliminating resistance trends, and lowering healthcare costs.
\end{abstract}

Keywords: Diabetic Foot Infection, Polymicrobial Infections, Amoxyclav

DOI: $10.7176 / \mathrm{JHMN} / 94-03$

Publication date:October $31^{\text {st }} 2021$

\section{Introduction}

Diabetes is a life-long condition. It affects many people all over the world. It is a significant public health issue [1]. About one-fourth of people with diabetes will develop an ulcer throughout their lifetime, and as many as half of those ulcers turns into infected [2, 3]. In human beings with diabetes and foot ulcers, numerous elements, such as inappropriate antibiotic treatment, the chronic nature of the wound, and frequent clinic admission, can have an effect on the presence of multidrug-resistant microorganisms inside the ulcer [4]. Moreover, the particular organisms identified in diabetic foot infections can differ not only from patient to patient and hospital to hospital but additionally from one a part of the country to some other [5].

Diabetic foot is a crippling condition. It offers long stretches of hospitalization. Health center prices may be very excessive and now and again not possible to undergo the charges, and the end result of an amputated limb. The ghostly limb adds insult to injury to the already battered psyche. No surprise, one of the most feared complications of diabetes is diabetic foot. The classic triad of neuropathy, ischemia, and infection characterizes diabetic foot. The first priority should be to prevent diabetic foot. This can be accomplished by identifying highrisk individuals, such as those who have peripheral neuropathy, peripheral vascular disease, foot deformities, or callu [6].

If not treated promptly, infectious microorganisms are linked to amputation of the infected foot, as well as an increase in hospital stay, cost of management, morbidity, and mortality [7]. Because most diabetic foot infections are true emergencies, antibiotic therapy should be initiated as soon as possible to improve the chances of saving the limb. Clinical presentation, gram-staining results, and knowledge of the organisms most commonly isolated from a specific infection should all be used to guide initial empirical therapy [8].

Diabetes-related foot ulcers are common and estimated to affect $15 \%$ of all diabetic individuals during their lifetime. Amputation is required in 15 to $20 \%$ of patients with such foot ulcers. Almost $85 \%$ of the amputations are preceded by diabetic foot ulcers [9-11]. Peripheral sensory neuropathy is the most important risk factor for the development of foot ulcers, followed by peripheral vascular disease. In diabetics, the proportion of neuropathic, neuroischemic, and purely ischemic lesions is 54,34, and 10\%, respectively [11]. It is estimated that approximately 40,000 legs are amputated in India each year, with 75 percent of them being neuropathic with secondary infection, which is potentially preventable. Barefoot walking, illiteracy, low socioeconomic status, late presentation by patients, primary care physician ignorance about diabetic foot care, and belief in alternative systems of medicine 
all contribute to this high prevalence [12].

Infection in a diabetic foot is a limb threatening condition because the consequences of deep infection in a diabetic foot are more disastrous than elsewhere mainly because of certain anatomical peculiarities. The foot has several compartments, which are inter-communicating and the infection can spread from one into another, and lack of pain allows the patient to continue ambulation further facilitating the spread. The foot also has soft tissues, which cannot resist infection, like plantar aponeurosis, tendons, muscle sheaths, and fascia. A combination of neuropathy, ischemia, and hyperglycemia worsens the situation by reducing the defense mechanism [6].

The correct antibiotic selection based on the antibiograms of isolates from diabetic foot infections is critical for the proper management of these infections. As a result, the current study sought to assess the bacteriology of diabetic foot ulcers at Hospital Geral de Palmas in Tocantins, Brazil, in order to determine the relative frequencies of bacterial isolates cultured from foot infections and to assess the isolated bacteria's in vitro antibiotic resistance and susceptibility to amoxyclav antibiotic.

\section{Materials and Methods \\ Materials \\ Study Design}

This cross-sectional study was designed to assess the bacterial profile cultured from the wounds of diabetic foot patients, and to assess the functional pattern of the amoxicillin antibiotic on the cultured microorganisms.

\section{Data Collection}

Laboratory data from May 2020 to May 2021 were routinely collected from the microbiology department from Bangladesh Institute of health and sciences hospital (BIHS) Dhaka, Bangladesh. The total sample volumes were 378.

\section{Methods}

\section{Swab sampling}

Wound beds were prepared before specimen collection, where the wound immediate surface exudates and contaminants were cleansed off with moistened sterile gauze and sterile normal saline solution. Dressed wounds were cleansed with non bacteriostatic sterile normal saline after removing the dressing. Aseptically the end of a sterile cotton-tipped applicator was rotated over $1 \mathrm{~cm}^{2}$ area for 5 seconds with sufficient pressure to express fluid and bacteria to surface from within the wound tissue [13]. The specimens were placed into sterile transport containers and sent to the microbiology laboratory for aerobic culturing within 20 minutes. Anaerobic culturing was not performed in this study $[14,15]$.

\section{Bacterial Culture}

Cultures were processed following the standard procedure [14,15]. Samples were inoculated on MacConkey agar (Oxoid), Chocolate agar (Oxoid) and Blood agar (Oxoid) media plate under class-II laminar airflow (NUVO SanajiMalzemelzeni, ImalatVcTicaret A.S, Turkey). The inoculum on the plate was streaked out for discrete colonies with a sterile wire loop sterilized by auto loop sterilizer (Germany) following standard procedures. The culture plates were incubated at $37^{\circ} \mathrm{C}$ by an incubator (Germany) for 48 hours and observed for the growth of bacteria through formation of colonies. All the bacteria were isolated and identified using morphological, microscopy (Japan) and biochemical tests like TSI (HiMedia), MIU (HiMedia) and Simmons Citrate (HiMedia) agar following standard procedures.

\section{Antibiotic Susceptibility Assessment}

The disc diffusion technique was used for antibacterial susceptibility testing of the isolates [16-18] using commercial antibiotics containing discs. We used the commercial antibiotic disc. Bacterial susceptibility was determined by the Kirby-Bauer disc diffusion method using antibiotic containing discs from Oxoid Ltd, UK. Amoxyclav antibiotic discs were used in this study. Interpretation of results was analysed using zone sizes. Zones of inhibition $\geq 21 \mathrm{~mm}$ will be considered sensitive, $16-20 \mathrm{~mm}$ intermediate and $<15 \mathrm{~mm}$ resistant. Isolates were classified as either sensitive or resistant based on the definition of the Clinical and Laboratory Standard Institute [17].

\section{Statistical Analysis}

Data were assessed by using the free software GNU PSPP stable release 1.4.1/ September 5, 2020. It has a graphical

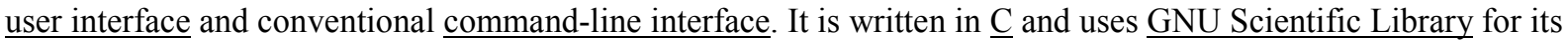
mathematical routines. The name has "no official acronymic expansion". Statistical frequencies were analyzed here. 


\section{Results}

A diabetic treatment center provided us with 378 participants for this research. They had all been diagnosed with diabetes and had diabetic feet. Males made up 247 (60.0\%) of the subjects, with 165 females (40.0\%) (Figure-1).

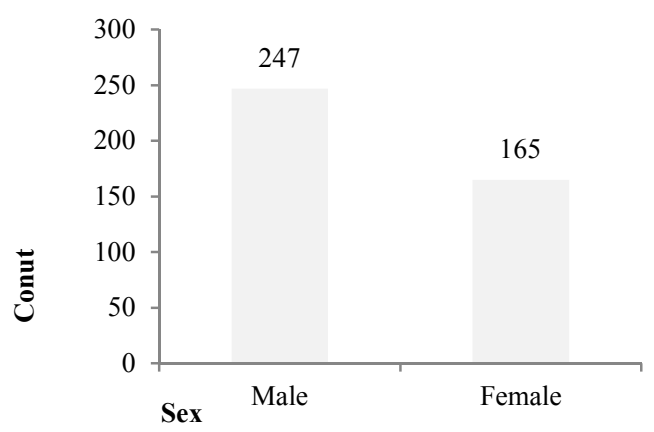

Figure-1: Sex distribution of the diabetic foot patients.

Among 378 diabetic foot subjects in the age group 10-20 years, males were $6(1.5 \%)$ and females were 1 $(0.2 \%)$. The age group $>20-30$ years showed that males were $2(0.5 \%)$ and females were $13(3.2 \%) .27(6.6 \%)$ males and $17(4.1 \%)$ females were found in the age group $>30-40$ years. The age group $>40-50$ years showed that almost equal males and females were in this area. Males were $55(13.3 \%)$ and females were $57(13.8 \%)$. The highest female subjects were found in

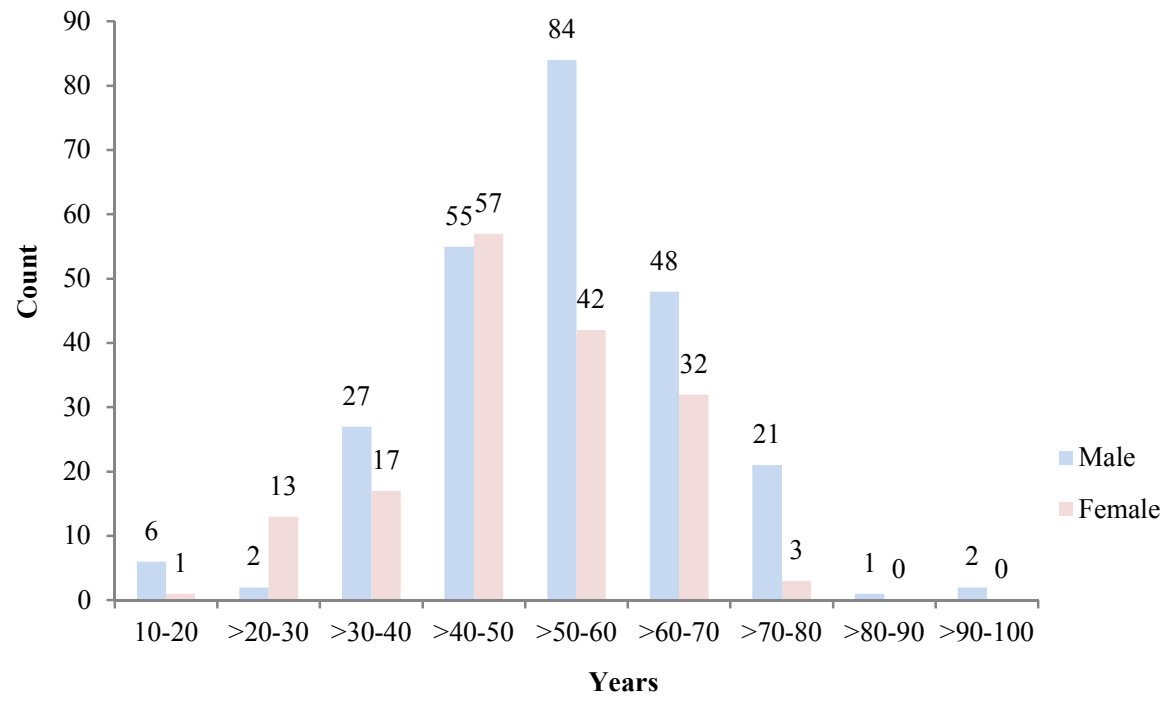

Figure-2: Sex distribution in different age groups.

the $>40-50$ year age group. The highest peak for diabetic foot subjects went to males, $84(20.4 \%)$ in the age group category $>50-60$ years and in this category females were $42(10.2 \%) .48$ males $(11.7 \%)$ and 32 females $(7.8 \%)$ were found in the age group $>60-70$ years. The age group $>70-80$ years was represented by 21 males $(5.1 \%)$ and 3 females $(0.7 \%)$. age groups $>80-90$ years and $>90-100$ years showed no female subjects. 1 male $(0.2 \%)$ was found in the age group $>80-90$ years and 2 males $(0.5 \%)$ were found in the age group $>90-100$ years (Figure-2). 
Table-1: Distribution of microorganisms among diabetic foot patients.

\begin{tabular}{|c|c|c|c|c|c|}
\hline Microorganism & & $\begin{array}{l}\text { Frequenc } \\
\mathrm{y}\end{array}$ & $\begin{array}{l}\text { Percen } \\
\mathbf{t}\end{array}$ & $\begin{array}{l}\text { Valid } \\
\text { Percent }\end{array}$ & $\begin{array}{l}\text { Cumulative } \\
\text { Percent }\end{array}$ \\
\hline Acinatobacter spp. & & 11 & 2.91 & 2.91 & 3.17 \\
\hline CONS (Staphylococcus & aureus and & & & & \\
\hline \multicolumn{6}{|l|}{ Staphylococcus } \\
\hline Saprophyticu) & & 10 & 2.65 & 2.65 & 5.82 \\
\hline Citrobacter Freundii & & 4 & 1.06 & 1.06 & 6.88 \\
\hline Citrobacter spp. & & 9 & 2.38 & 2.38 & 9.26 \\
\hline Enterobacter spp. & & 27 & 7.14 & 7.14 & 16.4 \\
\hline Enterococcus spp. & & 20 & 5.29 & 5.29 & 21.69 \\
\hline Escherichia coli & & 27 & 7.14 & 7.14 & 28.84 \\
\hline Klebsiella spp. & & 48 & 12.70 & 12.70 & 41.53 \\
\hline Proteus spp. & & 42 & 11.11 & 11.11 & 52.65 \\
\hline Proteus vulgaris & & 3 & 0.79 & 0.79 & 53.44 \\
\hline Pseudomonas aeruginosa & & 8 & 2.12 & 2.12 & 55.56 \\
\hline Pseudomonas spp. & & 44 & 11.64 & 11.64 & 67.2 \\
\hline Serratia marcescens & & 1 & 0.26 & 0.26 & 67.46 \\
\hline Staphylococcus aureus & & 123 & 32.54 & 32.54 & 100 \\
\hline Total & & 378 & 100 & 100 & \\
\hline
\end{tabular}

Among 378 diabetic foot patients (no missing data), 2.91\% were infected with Acinetobacter spp., 1.06\% were with Citrobacter freundii. 2.38\% were infected with Citrobacter spp., CoNS were in 2.65\% of patients. Enterobacter spp. was responsible for $7.14 \%$ of infections. Escherichia coli caused $7.14 \%$ of infections. $12.70 \%$ infection identified for Klebsiella spp. and $11.11 \%$ for Proteus spp.. Proteus vulgaris infected a small percentage of the patients and that was $0.79 \%$. Pseudomonas aeruginosa was dedicated to $2.12 \%$ infection. Among all microorganisms, the lowest infection was found by Serratia marcescens and the infection rate was $0.26 \%$. The highest number of infections were caused by Staphylococcus aureus. Here we found a 32.54\% infection rate (Table-1).

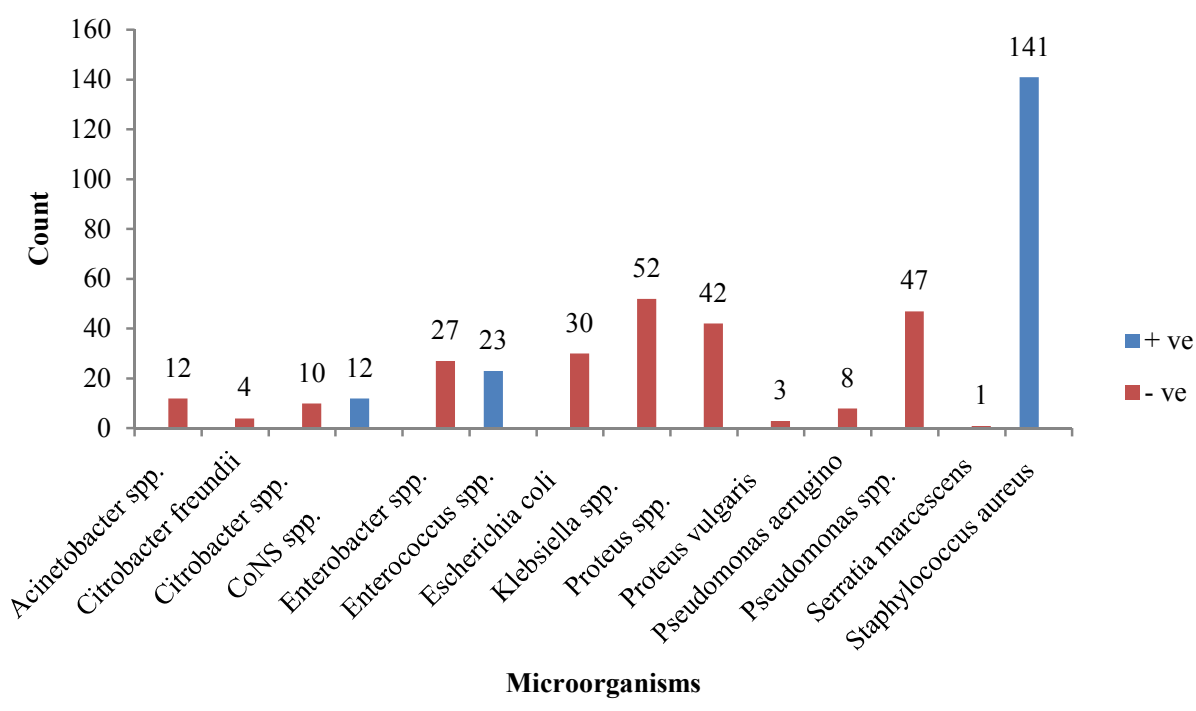

Figure-3: Distribution of gram positive (+ ve) and gram negative (-ve) microorganisms among diabetic foot patients.

Among the all microorganisms (14) highest of organisms were gram negative (10) and lowest numbers were gram positive (4).Acinetobacter spp. (12), Citrobacter freundii (4), Citrobacter spp. (10), Enterobacter spp. (27) Escherichia coli (30), Klebsiella spp. (52) Proteus spp. (42), Proteus vulgaris (3), Pseudomonas aeruginosa (8), and Pseudomonas spp. (47) were gram negative bacteria. 


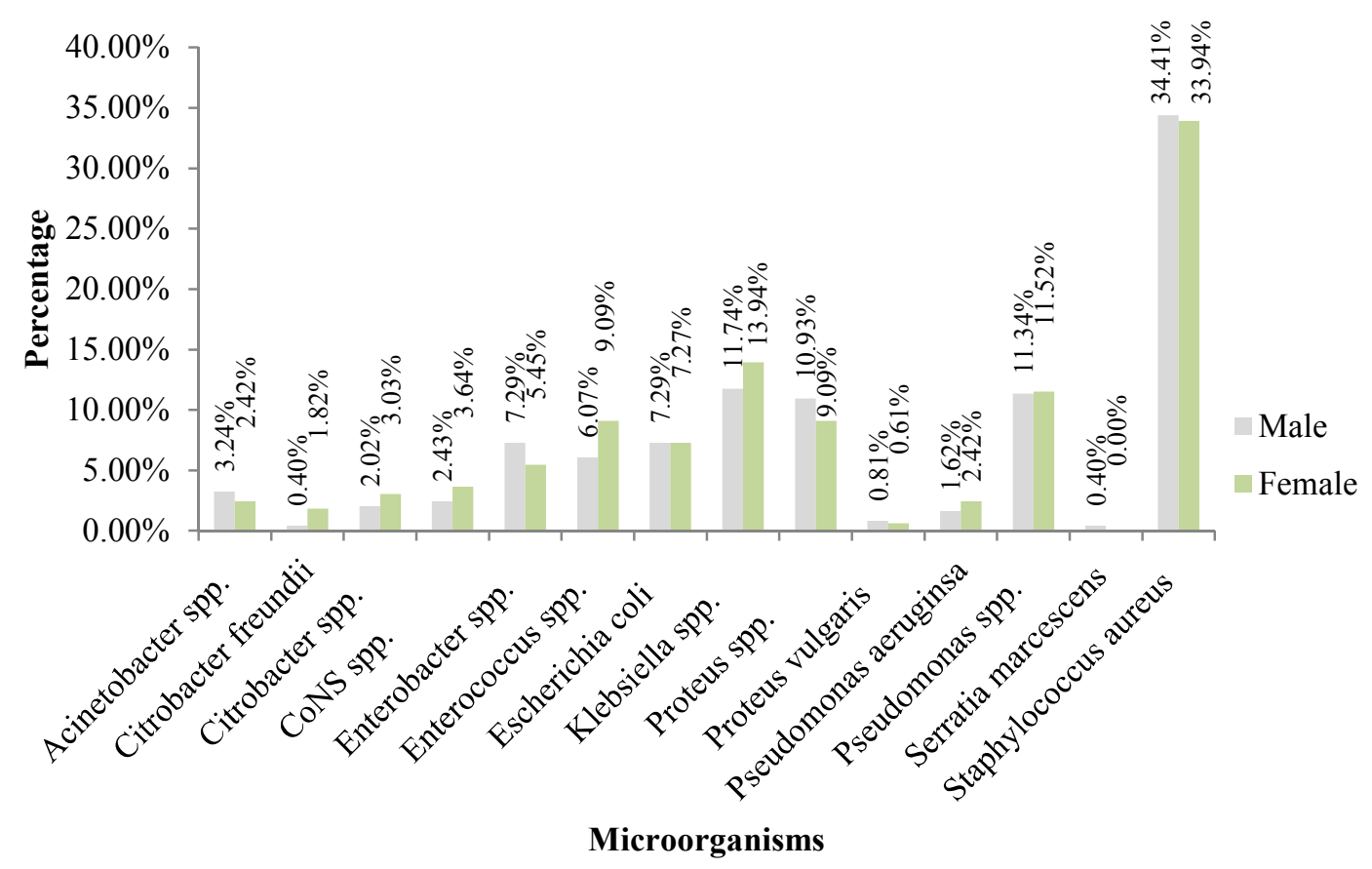

Figure-4: Sex distribution of gram positive (+ ve) and gram negative (-ve) microorganisms among diabetic foot patients.

Figure-4 described sex distribution regarding specific microorganism. i) In respect to Acinetobacter spp. pattern was $3.24 \%$ for male and $2.42 \%$ for female, ii) $0.01 \%$ male was found for Citrobacter feundii iii) $2.02 \%$ of males and $3.03 \%$ of females were found infected with Citrobacter spp. iv) $2.43 \%$ male was found for CoNS and female was 3.64\%. v) 7.29\% male was found for Enterobacter spp. and female was found 5.45\%. vi) $6.07 \%$ of males were infected with Enterococcus spp. and 9.09\% female. vii) Escherichia coli found in 7.29\% male, female percentage was 7.27. viii) Male found $11.74 \%$ and $13.94 \%$ female in respect to Klebsiella spp. ix) Proteus spp. was found in $10.93 \%$ male and in $9.09 \%$ female. $\mathrm{x}$ ) Proteus vulgaris was found in $0.81 \%$ of males and in $0.61 \%$ of females. xi) $1.62 \%$ of males were found with Pseudomonas aureginosa infection. $2.42 \%$ females were found with Pseudomonas aureginosa infection. xii. No female was found with Serratia marcescens and $100.00 \%$ were males. xiii. Staphylococcus aureus was found as a big name in our experiment. $34.41 \%$ of males were infected by this organism and the female percentage was 33.94 .

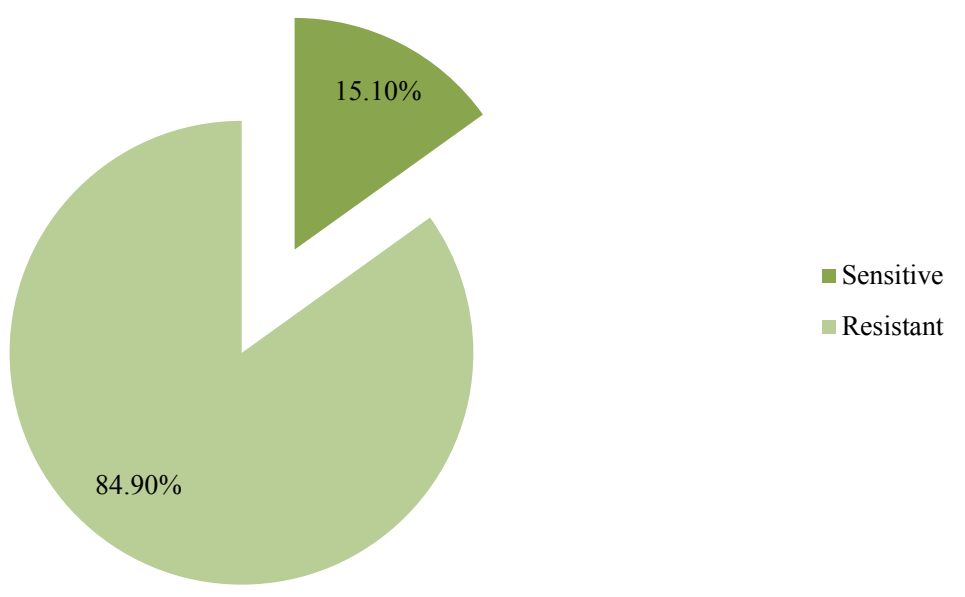

Figure-5: Amoxyclav's functional profile.

As a whole, we got a scenario that amoxyclav was sensitive for $15.10 \%$ of diabetic foot patients and $84.90 \%$ of patients found resistant (Figure-5). 


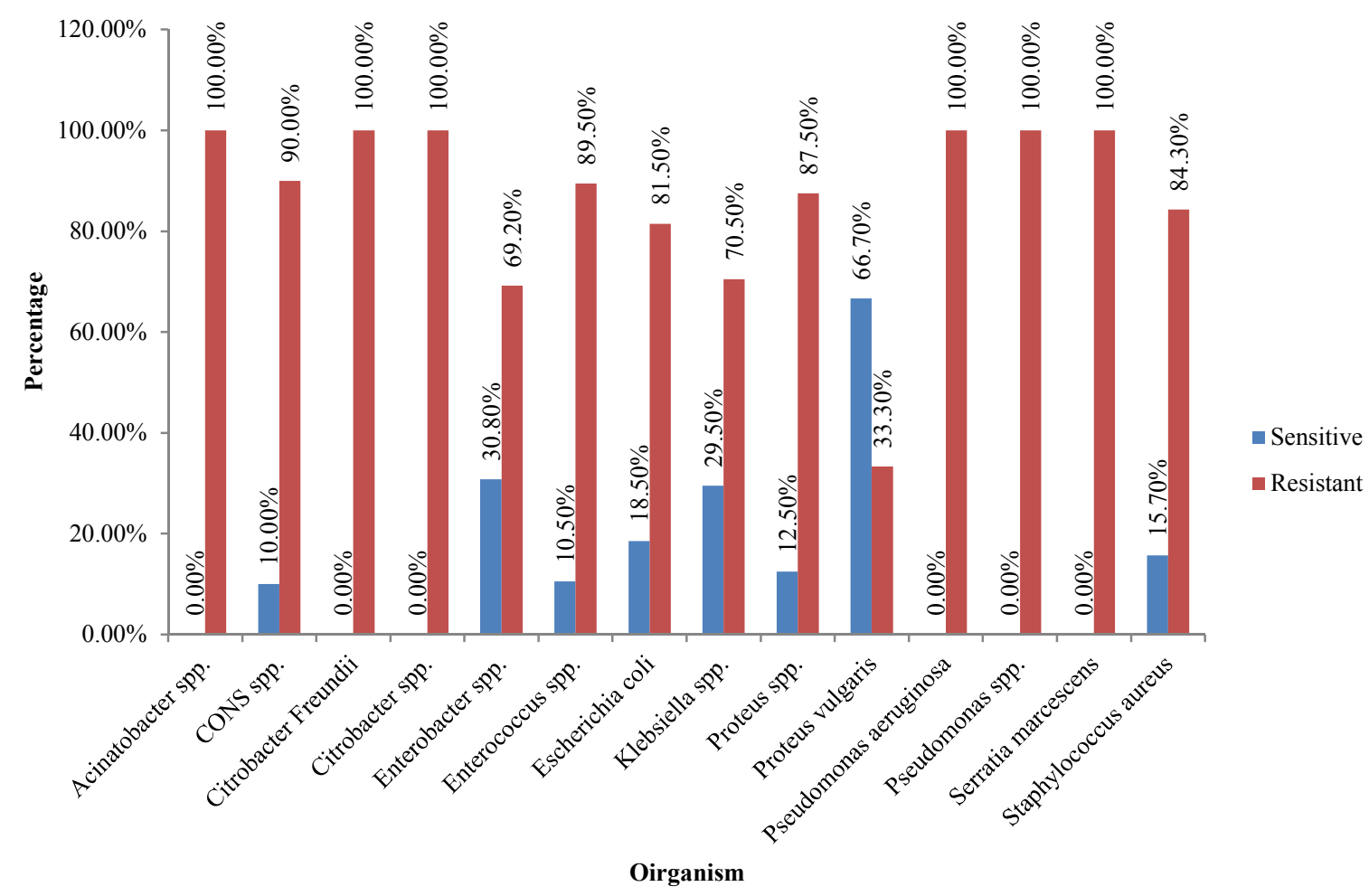

Figure-6: Sensitivity and resistant pattern of amoxyclav against different microorganisms.

Figure-2 described resistant and sensitivity pattern against amoxyclav. i) In respect to Acinetobacter spp. pattern was $0.00-100.00 \%$, that means $0.00 \%$ was for sensitivity and $100.00 \%$ was for resistant ii) $10.00 \%$ sensitivity was found for Citrobacter feundii and $90.00 \%$ for resistant iii) $100.00 \%$ sensitive applied for Citrobacter spp. iv) $100.00 \%$ sensitivity was found for CoNS spp. v) $30.80 \%$ sensitivity and $69.20 \%$ were found for Enterobacter spp. vi) $10.50 \%$ resistant Enterococcus spp. was found rest $89.50 \%$ was sensitive. vii) Escherichia coli showed $18.50 \%$ resistance against amoxyclav, sensitivity percentage looked high (89.50\%). viii) amoxyclav found $29.50 \%$ resistant and $70.50 \%$ sensitive against Klebsiella spp. ix) Proteus spp. was found $12.50 \%$ resistant and $87.50 \%$ sensitive. x) Proteus vulgaris infection, $33.30 \%$ diabetic foot infection with this organism showed resistance against amoxyclav and $66.70 \%$ were fine with amoxyclav. xi) $100.00 \%$ diabetic foot infection with this organism showed resistance against amoxyclav. xii) Serratia marcescens showed 100.00\% resistant. xiii) Staphylococcus aureus was found as a big name in our experiment. $15.70 \%$ of this organism showed resistance against target antibiotic and $84.30 \%$ showed sensitive.

\section{Discussion}

Foot infections in patients with diabetes are a common, complex, and costly problem [19]. In the present study, we found that elderly patients ( $>60$ years of age) constituted the majority of patients with foot infections. This may be explained by the fact that foot lesions occur commonly among patients with diabetes, particularly the elderly and those with sensory neuropathy [20]. Previous studies have shown that the susceptibility to foot infections is greater in male patients than in female patients $[1,5]$. However, in our study, we did not find differences between male and female patients, which may be because of the limited number of patients.

Diabetic foot ulcers are colonized by pathogenic bacteria that may predispose a susceptible patient to a lower extremity infection, defined as the invasion and multiplication of microorganisms in body tissues associated with tissue destruction or host inflammatory responses [21]. In the present study, we found that the majority of lesions were located on the right toe and plantar region, and varied in duration from 1 day to more than 90 days. Additionally, recent lesions (1-30 days) were the most common. Our findings are in accordance with the results of Donoso et al. (2013) [22].

This study is limited by the fact that cultures for anaerobic bacteria could not be performed. In diabetic foot infections, the role of anaerobic bacteria is particularly unclear; some studies have reported that anaerobic bacteria play a minor role $[23,24]$, while other studies found a high incidence of anaerobic bacteria $[5,25]$.

The most common pathogens isolated were Gram-positive cocci, such as Staphylococcus aureus and Staphylococcus saprophyticus (CONS) and Gram-negative rods, such as Proteus spp. and Enterobacter spp. Although the findings of our study are consistent with the results of previous studies showing that Gram-positive 
bacteria were predominant in diabetic foot infections [25], other studies have reported that Gram-negative bacteria were predominant in particular regions $[26,27]$. These results suggest, in part, differences in the type and severity of infections [5, 28]. Aerobic Gram-negative bacteria (mainly Enterobacteriaceae and sometimes Pseudomonas aeruginosa or other Gram-negative species) are usually isolated in conjunction with Gram-positive cocci in patients with chronic or previously treated infections [26], which is consistent with our findings. Polymicrobial infections accounted for $70 \%$ of all infections. Although polymicrobial etiology has been implicated in diabetic foot infections [28], a previous study reported the predominance of monomicrobial infections [28]. These discrepancies suggest differences in diabetic foot infections, with severe infections usually having polymicrobial isolates and mild infections usually having monomicrobial isolates [28, 29].

\section{Conclusions}

The present study report has some limitations because cultures for anaerobic bacteria could not be performed and sample size was small. However, it confirmed the high prevalence of multidrug-resistant pathogens in diabetic foot ulcers. Diabetic foot infections were predominantly due to Gram-positive bacteria, such as Staphylococcus aureus, or were polymicrobial infections. Many studies on the bacteriology of diabetic foot infections have reported results that vary and are often contradictory $[25,26,28]$. In such cases, application of molecular techniques may lead to more accurate microbial characterizations and targeted antibiotic therapy. Therefore, it is necessary to evaluate the different microorganisms infecting the wound on a routine basis and to know the antibiotic susceptibility patterns of the isolates from the infected wound in patients with diabetic foot lesions. This knowledge is crucial for planning the treatment of these patients with the appropriate antibiotics, reducing resistance patterns, and minimizing healthcare costs. We hope the data presented on this article can assist the clinicians in determining the multidrug-resistant pathogens in diabetic foot ulcers. In this study amoxyclav was found sensitive for $15.10 \%$ of diabetic foot patients and $84.90 \%$ of patients were found resistant.

\section{References}

1. Sivanmaliappan, T.S. and M. Sevanan, Antimicrobial susceptibility patterns of Pseudomonas aeruginosa from diabetes patients with foot ulcers. International journal of microbiology, 2011. 2011.

2. $\quad$ Lipsky, B.A., et al., Diagnosis and treatment of diabetic foot infections. Clinical infectious diseases, 2004: p. $885-910$

3. Lavery, L.A., et al., Validation of the Infectious Diseases Society of America's diabetic foot infection classification system. Clinical infectious diseases, 2007. 44(4): p. 562-565.

4. Kandemir, Ö., et al., Risk factors for infection of the diabetic foot with multi-antibiotic resistant microorganisms. Journal of Infection, 2007. 54(5): p. 439-445.

5. $\quad$ El-Tahawy, A.T., Bacteriology of diabetic foot. Saudi medical journal, 2000. 21(4): p. 344-347.

6. Pendsey, S.P., Understanding diabetic foot. International journal of diabetes in developing countries, 2010. 30(2): p. 75.

7. Hartemann - Heurtier, A., et al., Diabetic foot ulcer and multidrug - resistant organisms: risk factors and impact. Diabetic Medicine, 2004. 21(7): p. 710-715.

8. Gentry, L.O., Diagnosis and management of the diabetic foot ulcer. Journal of Antimicrobial Chemotherapy, 1993. 32(suppl A): p. 77-89.

9. $\quad$ Palumbo, P. and L.J. Melton, Peripheral vascular disease and diabetes. Diabetes in America, 1985. 2: $\mathrm{p}$. 401-408.

10. Pendsey, S., Diabetic foot: a clinical atlas. 2013: CRC Press.

11. Pecoraro, R.E., G.E. Reiber, and E.M. Burgess, Pathways to diabetic limb amputation: basis for prevention. Diabetes care, 1990. 13(5): p. 513-521.

12. Pendsey, S. and Z.G. Abbas, The step-by-step program for reducing diabetic foot problems: a model for the developing world. Current Diabetes Reports, 2007. 7(6): p. 425-428.

13. Levine, N.S., et al., The quantitative swab culture and smear: A quick, simple method for determining the number of viable aerobic bacteria on open wounds. The Journal of trauma, 1976. 16(2): p. 89-94.

14. York, M., Quantitative cultures of wound tissues. Clinical microbiology procedures handbook, 2004. 1: p. 2.1-3.13.

15. Reisner, B. and G. Woods, Specimen processing. Murray PR. eds. Manual of Clinical Microbiology 7. th edition. 1999, ASM Press Washington DC.

16. Baur, A., W. Kirby, and J. Sherris, Turck. Antibiotic susceptibility testing by a standardized single disc method. The American Journal of Clinical Pathology, 1966. 36: p. 493-496.

17. Wayne, P., Clinical and laboratory standards institute; 2007. Performance standards for antimicrobial susceptibility testing. CLSI document M100-S17, 2005.

18. Nwanze, P., et al., Urinary tract infection in Okada village: Prevalence and antimicrobial susceptibility pattern. Scientific Research and Essay, 2007. 2(4): p. 112-116. 
19. Lipsky, B.A., A report from the international consensus on diagnosing and treating the infected diabetic foot. Diabetes/metabolism research and reviews, 2004. 20(S1): p. S68-S77.

20. Lipsky, B.A., R.E. Pecoraro, and J.H. Ahroni, Foot ulceration and infections in elderly diabetics. Clinics in geriatric medicine, 1990. 6(4): p. 747-769.

21. Hobizal, K.B. and D.K. Wukich, Diabetic foot infections: current concept review. Diabetic foot \& ankle, 2012. 3(1): p. 18409.

22. Donoso, M.T.V., E.G. Rosa, and E.L. Borges, Perfil dos pacientes com pé diabético de um serviço público de saúde. Revista de Enfermagem UFPE on line, 2013. 7(7): p. 4740-4746.

23. Senneville, E., et al., Culture of percutaneous bone biopsy specimens for diagnosis of diabetic foot osteomyelitis: concordance with ulcer swab cultures. Clinical infectious diseases, 2006. 42(1): p. 57-62.

24. González, F.C., et al., Infections in diabetic foot ulcers. European journal of internal medicine, 2003. 14(5): p. 341-343.

25. Abdulrazak, A., et al., Bacteriological study of diabetic foot infections. Journal of diabetes and its complications, 2005. 19(3): p. 138-141.

26. Gadepalli, R., et al., A clinico-microbiological study of diabetic foot ulcers in an Indian tertiary care hospital. Diabetes care, 2006. 29(8): p. 1727-1732.

27. Singh, S.K., et al., Detecting aerobic bacterial diversity in patients with diabetic foot wounds using ERICPCR: a preliminary communication. The international journal of lower extremity wounds, 2009. 8(4): p. 203-208.

28. Citron, D.M., et al., Bacteriology of moderate-to-severe diabetic foot infections and in vitro activity of antimicrobial agents. Journal of clinical Microbiology, 2007. 45(9): p. 2819-2828.

29. Anandi, C., et al., Bacteriology of diabetic foot lesions. Indian journal of medical microbiology, 2004. 22(3): p. 175-178. 